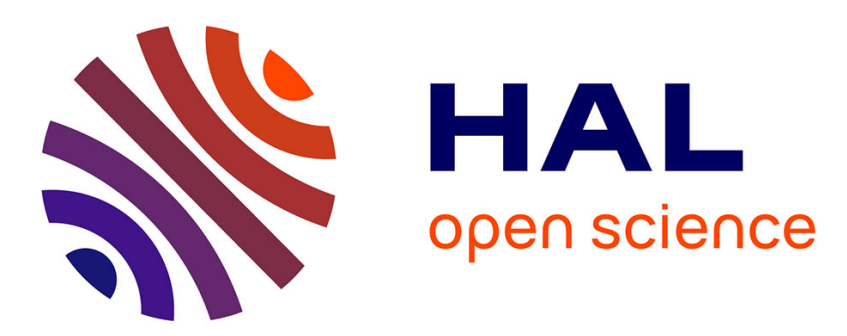

\title{
Systèmes informatiques d'aide à la décision en médecine: panorama des approches utilisant les données et les connaissances
}

Brigitte Séroussi, Jacques Bouaud

\section{- To cite this version:}

Brigitte Séroussi, Jacques Bouaud. Systèmes informatiques d'aide à la décision en médecine: panorama des approches utilisant les données et les connaissances. Pratique Neurologique - FMC, 2014, 5 (4), pp.303-316. 10.1016/j.praneu.2014.09.006 . hal-01100249

\section{HAL Id: hal-01100249 \\ https://hal.sorbonne-universite.fr/hal-01100249}

Submitted on 7 Jan 2015

HAL is a multi-disciplinary open access archive for the deposit and dissemination of scientific research documents, whether they are published or not. The documents may come from teaching and research institutions in France or abroad, or from public or private research centers.
L'archive ouverte pluridisciplinaire HAL, est destinée au dépôt et à la diffusion de documents scientifiques de niveau recherche, publiés ou non, émanant des établissements d'enseignement et de recherche français ou étrangers, des laboratoires publics ou privés. 


\title{
Systèmes informatiques d'aide à la décision en médecine: panorama des approches utilisant les données et les connaissances
}

\author{
Computerized decision support systems: overview of data- and knowledge-based \\ approaches
}

\author{
Brigitte Séroussi $^{1,2}$, Jacques Bouaud $^{3,1}$ \\ ${ }^{1}$ Sorbonne Universités, UPMC Université Paris 06, UMR_S 1142, LIMICS, Paris, France ; INSERM, U1142, \\ LIMICS, Paris, France ; Université Paris 13, Sorbonne Paris Cité, LIMICS, (UMR_S 1142), Bobigny, France \\ ${ }^{2}$ AP-HP, Hôpital Tenon, DSP, Paris, France; APREC, Paris, France \\ ${ }^{3}$ AP-HP, DRCD, Paris, France
}

\begin{abstract}
Résumé
Les systèmes d'aide à la décision médicale (SADM) sont des outils informatiques capables de traiter l'ensemble des caractéristiques d'un patient donné afin de générer les diagnostics probables de son état clinique (aide au diagnostic) ou les traitements qui lui seraient adaptés (aide à la thérapeutique). Le terme SADM recouvre aujourd'hui un ensemble d'outils variés, plus ou moins complexes. Cet article dresse un panorama de ces différents outils et des approches qui en sous-tendent le fonctionnement. Les approches numériques se fondent essentiellement sur l'analyse de jeux de données. Elles ont initialement été appliquées à l'aide au diagnostic. Du fait du déploiement des dossiers patients informatisés, on dispose aujourd'hui de nombreuses données utilisées par les modèles prédictifs d'aide à la thérapeutique. Les approches symboliques utilisent une modélisation les connaissances mobilisées par le raisonnement pour décider. Elles ont été initialement mises en œuvre avec les systèmes experts qui visaient à reproduire le raisonnement des experts cliniciens engagés dans une démarche décisionnelle. Récemment, conformément au principe de la médecine fondée sur les données probantes, l'expertise subjective des systèmes experts a été remplacée par les connaissances objectives des recommandations de bonne pratique dans les SADM modernes. Ces systèmes, qu'ils soient historiques ou actuels, sont décrits, les différents types d'exploitation des connaissances qu'ils proposent, que ce soit en mode automatique ou documentaire, sont présentés. Longtemps cantonnés aux laboratoires de recherche et aux expérimentations pilotes, il est probable que, compte tenu des exigences sociétales en terme de qualité des soins, les SADM soient dans un futur proche appelés à être utilisés en routine afin d'orienter les pratiques et d'évaluer les performances.
\end{abstract}

\begin{abstract}
Clinical decision support systems (CDSSs) are computerized tools capable of handling all the characteristics of a given patient to provide her most probable diagnoses (diagnostic decision-making) or best treatments (therapeutic decision-making). CDSSs today cover a wide range of tools of different purposes and complexities. This article provides an overview of CDSSs and of the different approaches implemented. Numerical approaches are mainly based on the analysis of data sets. They were initially applied to diagnosis decision support. Due to the deployment of computerized patient records, many data became available and used by predictive models to support therapeutic decision-making. Symbolic approaches rely on the modelling of the knowledge used for decision. These approaches were originally implemented for expert systems expected to reproduce the reasoning of expert clinicians engaged in a decision-making process. Recently, following the principle of evidence-based medicine, the subjective expertise of expert systems has been replaced by the objective knowledge of clinical practice guidelines in modern CDSSs. Different CDSSs, whether historical or current, are described, various types of knowledge exploitation, either in automatic mode or documentary, are presented. Used to be confined to research laboratories and pilot experiments, it is likely that, given the demands of modern societies for care quality, CDSSs are called to be routinely used to guide clinical practices and evaluate performance in the near future.
\end{abstract}


Mots-clés :Techniques d'aide à la décision, Systèmes d'aide à la décision clinique, Prise de décision assistée par ordinateur

Keywords:Decision support techniques,Clinical decision support systems, Computer-assisted decision making

\section{Introduction}

Du fait de l'accroissement continu des connaissances médicales, les signes, les symptômes et les maladies se sont spécialisés, les investigations complémentaires se sont multipliées, de nombreuses nouvelles molécules sont arrivées sur le marché. Les médecins, qu'ils soient généralistes ou spécialistes, ne peuvent plus maîtriser l'ensemble du savoir médical permettant de reconnaître les maladies ou de déterminer la meilleure prise en charge thérapeutique. Aussi, ils ont souvent recours à des sources d'information externes, traditionnellement les collègues, les livres, et la littérature scientifique, pour trouver les informations qui leur manquent. Néanmoins, en dépit (ou à cause ?) de la diffusion en ligne de grands volumes de ressources documentaires facilement accessibles, la recherche de la solution au problème posé par un patient donné est une tâche difficile. Très tôt, les qualités de l'ordinateur (mémoire, rapidité, puissance de calcul) se sont imposées comme des solutions potentielles à cette difficulté et des systèmes informatiques d'aide à la décision médicale ont été développés.

Les systèmes d'aide à la décision médicale (SADM) sont définis de manière très générale comme des outilsinformatiques « dont le but est de fournir aux cliniciens en temps et lieux utiles les informations décrivant la situation clinique d'un patient ainsi que les connaissances appropriées à cette situation, correctement filtrées et présentées afin d'améliorer la qualité des soins et la santé des patients. » (Berner, 2009). Il existe ainsi des SADM pour l'ensemble des activités médicales (prévention, dépistage, diagnostic, traitement) et la majorité des spécialités médicales (maladies chroniques ou affections aiguës). Ces systèmes proposent des services pour les différentes catégories de médecins (généralistes, spécialistes, étudiants) et les différents modes d'exercice (cabinets médicaux, hôpitaux, services d'urgence ou de réanimation). Plus récemment, des SADMont été développés à destination des patients afin qu'ils soient mieux informés sur leur maladie et les soins qui pourraient leur êtreproposés dans un objectif de décision partagée.

L'objectif de cet article est de présenter les fondements des différentes approches d'aide à la décision médicale et de dresser un panorama des outils qui ont vocation à «entourer» les pratiques dans un futur proche. Notre propos se limitera aux systèmes d'aide à la décision diagnostique et aux systèmes d'aide à la décision thérapeutique. Ainsi, on ne traitera pas le cas des «logiciels d'aide à la prescription » (LAP) qui sont des outils de sécurisation de l'ordonnance. Contrairement aux systèmes d'aide à la décision thérapeutique qui permettent de déterminer le meilleur traitement pour un patient donné, les LAP procèdent uniquement à des vérifications syntaxiques au sein de l'ordonnance sur la base des caractéristiques des produits prescrits pour détecter les interactions médicamenteuses et contre-indications de certaines drogues. Ils permettent par exemple de prescrire un antibiotique pour une migraine, mais l'antibiotique choisi sera à la bonne dose, au bon rythme et sans interaction médicamenteuse avec les autres médicaments de l'ordonnance.

Parmi les différents types de SADM, on distingue classiquementles approches numériques de l'aide à la décision qui se fondent surdes données et les approches symboliques de l'aide à la décision qui utilisent des connaissances. Dans le premier cas, les SADM s'appuient sur des modélisations mathématiques permettant de produire des probabilités à partir d'un jeu de données(probabilité d'un diagnostic, de la survenue d'un événement grave, etc.). On considérera également le cas des SADM permettant le calcul de scores. Dans le second cas, les SADM mettent en œuvre un raisonnement logique pour résoudre le problème posé par un patient donné qu'il soit de nature diagnostique ou thérapeutique. Le tableau 1 résume ces différentes approches. 
Tableau 1: $\quad$ Panorama des approches utilisées pour l'aide à la décision diagnostique et thérapeutique

\begin{tabular}{|l|ll|l|}
\hline & \multicolumn{1}{|c|}{ Aide au diagnostic } & \multicolumn{1}{c|}{ Aide à la thérapeutique } \\
\hline Approches numériques & $\bullet \begin{array}{l}\text { Modèles probabilistes } \\
\text { Calcul de scores }\end{array}$ & $\bullet$ Modèles pronostiques (Adjuvant! Online) \\
\hline Approches symboliques & $\bullet$ & Systèmes experts (DXplain) & $\begin{array}{l}\text { Systèmes experts (Mycin) } \\
\text { Approches documentaires et } \\
\text { automatiques pour la mise en ouvre des } \\
\text { recommandations de pratique clinique }\end{array}$ \\
\hline
\end{tabular}

\section{Décider à partir des données}

Historiquement, les premiers SADM étaient des systèmes d'aide au diagnostic. Ils utilisaient des approches numériques, essentiellement statistiques et probabilistes.

\subsection{Les approches probabilistes pour l'aide au diagnostic}

Les approches numériques proposent une modélisation commune des données du problème à résoudre. On considère une base de données construite à partir du recueil de kvariables (les signes, les symptômes, les résultats d'examens complémentaires) pour une cohorte de patients pour lesquels on dispose des diagnostics répartis en $\mathrm{n}$ catégories. Les approches probabilistes, développées à partir du théorème de Bayes, permettent de calculer pour tout nouveau patient caractérisé par un vecteur $\mathrm{X}$ des $k$ variables, les probabilités a posteriori des $\mathrm{n}$ différentes hypothèses diagnostiques $D_{i}$ :

$$
\forall i=1, \ldots n, P\left(D_{i} / X\right)=\frac{P\left(X / D_{i}\right) \cdot P\left(D_{i}\right)}{P(X)}
$$

Les $\mathrm{P}\left(\mathrm{D}_{\mathrm{i}}\right)$ qui représentent la prévalence des différents diagnostics et les $\mathrm{P}\left(\mathrm{X} / \mathrm{D}_{\mathrm{i}}\right)$, qui représentent les probabilités a priori des associations $\mathrm{X}$ de variables dans les diagnostics $\mathrm{D}_{\mathrm{i}}$ sont estimées par les fréquences correspondantes calculées sur la base de données. Parmi les premiers systèmes probabilistes, il faut retenir celui de de Dombal, développéau début des années 70, qui proposait une aide au diagnostic des douleurs aiguës de l'abdomen avec huit maladies et 50 signes, avecune performance de $91,8 \%$, significativement supérieure à celle des experts humains du domaine mesurée à 79,6\% (de Dombal et al., 1972). Toutefois, avec 19 diagnostics, les performances du système étaient significativement diminuées,avec seulement $69,9 \%$ de bons diagnostics(Wilson et al.,1977). Par ailleurs, utilisé dans un autre lieu que celui de sa conception, le système n'a pas fait la preuve de performances comparables et a nécessité un reparamétrage spécifique de la base de données pour estimer les probabilités apparaissant dans le modèle. Enfin, la production des résultats limitée à l'affichage d'une liste de probabilités, sans explication, en mode «boîte noire» (cf. figure 1), n'a pasétéjugée satisfaisante dans les situations où les cliniciens utilisateurs n'étaient pas d'accord avec le système. 


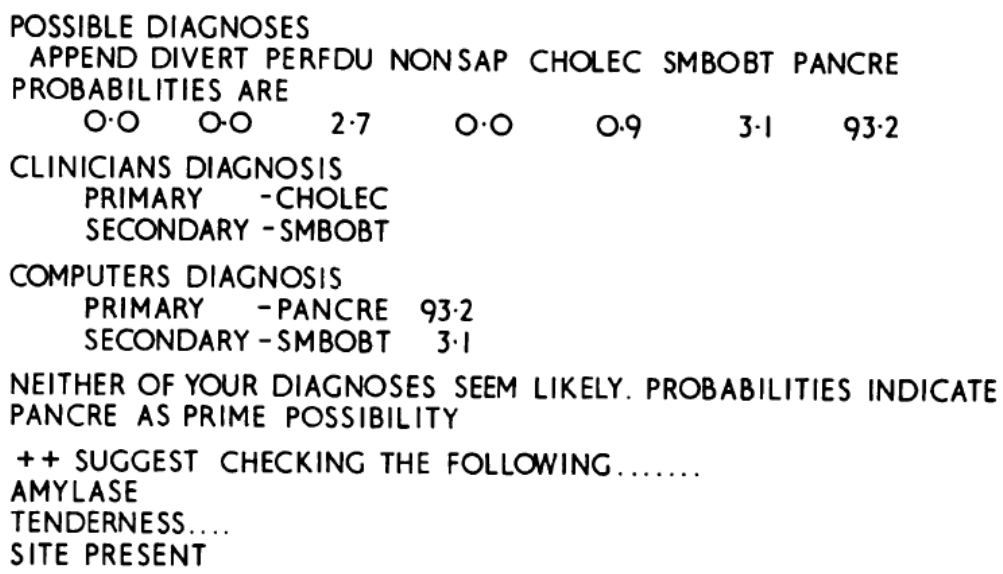

Figure 1: $\quad$ Interface du système d'aide au diagnostic des douleurs aiguës de l'abdomen (extrait de Horrocks et al., 1972).

\subsection{Les modèles pronostiques pour l'aide à la thérapeutique}

La médecine prédictive désigne l'ensemble des méthodes de prédiction statistique du délai de survenue d'un événement qui peut être l'apparition ou la récidive d'une maladie, l'apparition de métastases, le décès. L'objectif est de stratifier l'ensemble de la population en fonction du risque de survenue de l'événement et d'identifier les patients qui doivent bénéficier d'une prévention ciblée ou d'un traitement spécifique (intensification des traitements dans les groupes à haut risque, ou désescalade thérapeutique dans les groupes à bas risque). Dans l'idéal, la médecine prédictive permettrait de proposer des traitements «à la carte».

Un modèle pronostique utilise une modélisation mathématique de l'association entre un événement clinique représenté par une variable (variable à expliquer) et plusieurs caractéristiques dites variables explicatives. Les principaux modèles mathématiques sont les modèles de régression (régression logistique ou modèle de Cox).

Ce type d'approche a été utilisé en particulier avec le système Adjuvant! Online ${ }^{1}$ (Radvin et al., 2001) pour prédire la réponse aux traitements en cancérologie. La figure 2 illustre la visualisation graphique des probabilités de survie à 10 ans pour différents traitements du cancer du sein associant selon le cas chimiothérapie et/ou hormonothérapie, à partir d'un certain nombre de caractéristiques de la patiente (âge, état général, statut des récepteurs aux estrogènes, grade histologique, taille de la tumeur, envahissement ganglionnaire et type de la chimiothérapie envisagée).

\footnotetext{
${ }^{1}$ https://www.adjuvantonline.com/
} 


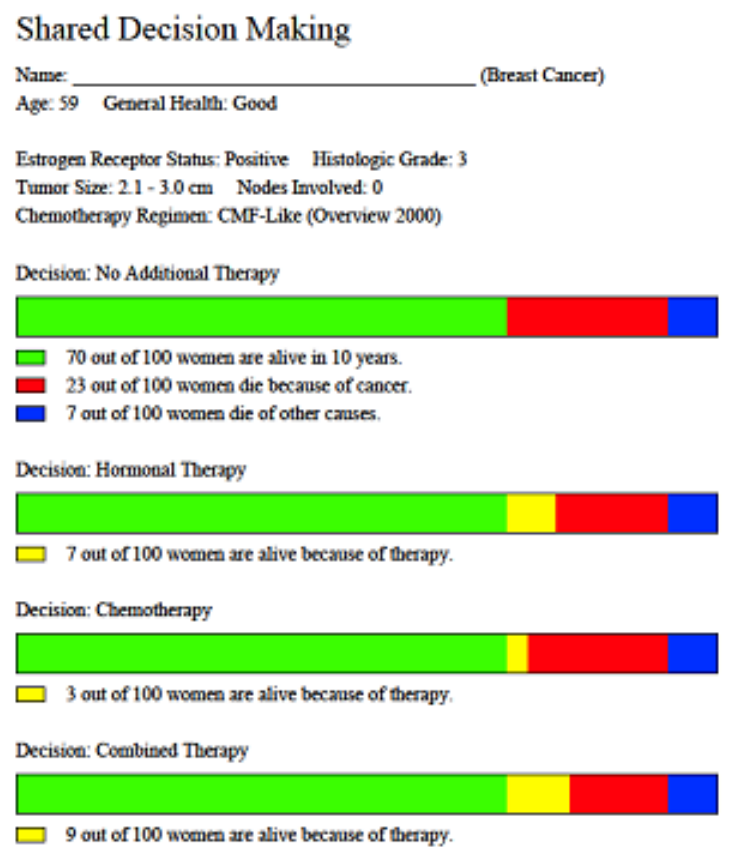

Figure 2: $\quad$ Interface du système Adjuvant! Online pour évaluer la survie à 10 ans dans les différents scénarios de traitement (sans traitement adjuvant, avec hormonothérapie seulement, avec chimiothérapie seulement, avec hormonothérapie et chimiothérapie).

Plus récemment, du fait du déploiement des dossiers médicaux informatisés, de la disponibilité de données structurées et de leur exploitation dans des entrepôts de données, il est possible de personnaliser le calcul de différents risques afin de profiler plus finement les patients. Une application de ce type d'approche est utilisée chez Kaiser Permanente aux Etats-Unis avec le système IndiGO (Individualized Guidelines and Outcomes) (Bellows et al., 2014).Commercialisé par la société Archimedes², le systèmepermet d'évaluer le risque de développer un diabète, un asthme, une maladie cardiaque et un certain nombre d'autres maladies chroniques. La figure 3 illustre une interface IndiGO avec les probabilités de survenue d'un AVC selon la mise en œuvre de certaines actions (arrêt du tabac, perte de poids, traitement médicamenteux par statine, etc.) chez un patient de 51 ans, diabétique, tabagique, en surpoids et sous inhibiteur de l'enzyme de conversion (informations extraites du dossier patient, apparaissant sur la fenêtre de gauche).

\footnotetext{
${ }^{2}$ http://archimedesmodel.com/
} 


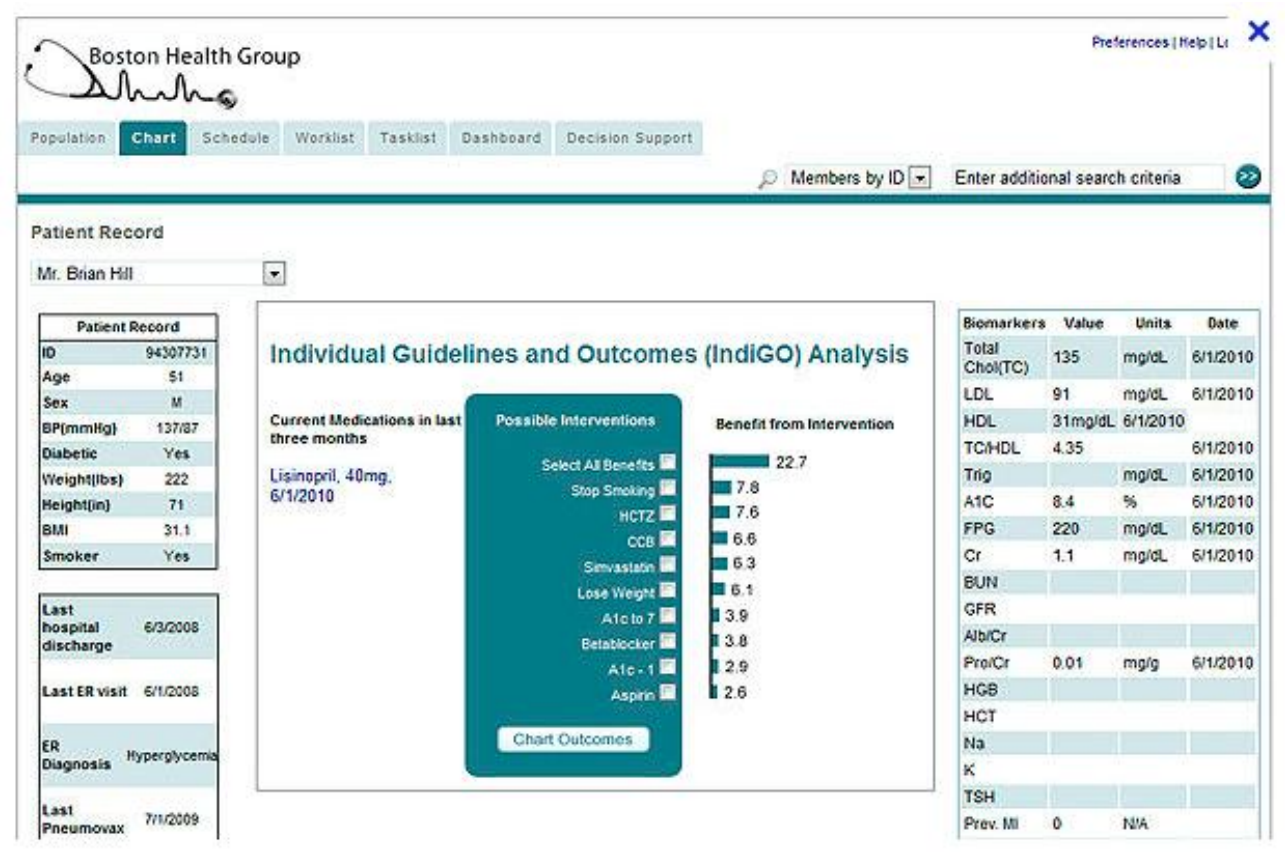

Figure 3: $\quad$ Interface IndiGO avec affichage des bénéfices associés à différentes interventions pour abaisser le risque d'AVC (consultation du site

http://www.medicalexpo.es/prod/archimedes/softwares-medicos-gestion-riesgos-enfermedades-81708535035.html le 27 mai 2014).

\subsection{L'aide au calcul de scores}

De très nombreux scores cliniques ont été construits et validés pour aider le médecin dans sa démarche diagnostique et thérapeutique. Certains sont connus de la plupart des médecins comme le Mini Mental State (MMS) qui est un test de référence dans le dépistage des démences ou le test de Fagerström qui permet d'évaluer la dépendance tabagique.

Le principe de ces scores est simple : le médecin pose un nombre fixe de questions standardisées et le score final prend souvent la forme d'une somme, pondérée ou non, des résultats des réponses aux questions. Des seuils permettent de conclure (par exemple : détérioration intellectuelle absente, légère, importante).

Dans le cas du MMS (cf. figure 4), le médecin pose au patient trente questions standardisées et compte les réponses justes. L'implémentation informatique du MMS le rend plus facile à utiliser en consultation. Le médecin n'a plus qu'à cliquer sur les cases qui conviennent, le score total est alors automatiquement calculé. 


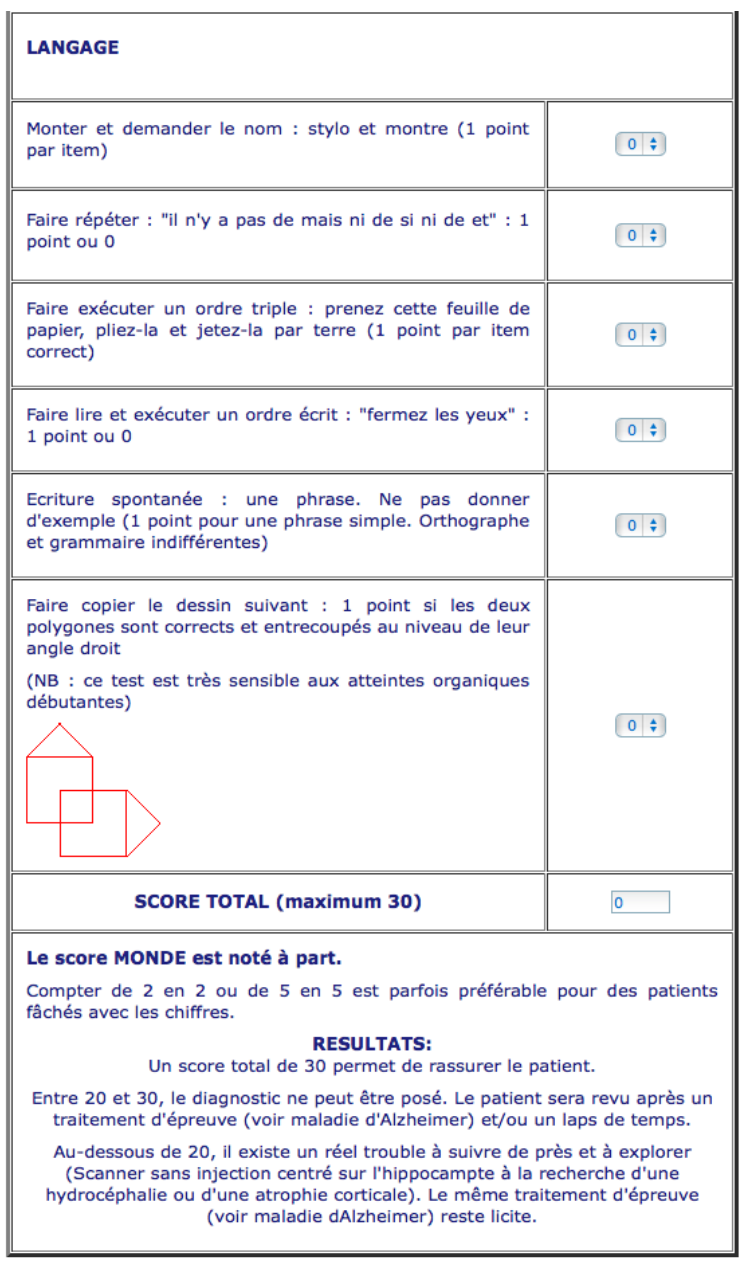

Figure 4: $\quad$ Extrait du Mini Mental Score (consultation du site http://www.telesantepaysdelaloire.fr/ le 27 mai 2014)

Les approches numériques de l'aide à la décision médicale basées sur les données sont toujours largement utilisées (en particulier dans le cas de la médecine prédictive et du calcul de score) et elles le seront sans doute de plus en plus du fait de l'accumulation de données de plus en plus structurées dans les dossiers patients informatisés. Néanmoins, la question de la validité des modèles au travers des données utilisées pour les calibrer reste entière. Il est à noter par ailleurs que l'aide à la décision dans ce cas, ne s'appuie pas sur un raisonnement qui proposerait une décision. Au contraire, les systèmes sont utilisés comme de super calculateurs de risques, de scores, autant de résultats qui viennent alimenter la réflexion du médecin qui garde le contrôle de la décision, et de la démarche décisionnelle.

\section{Décider à partir des connaissances}

Au cours des années 70, avec l'avènement de l'intelligence artificielle dont l'objectif était la recherche de moyens susceptibles de doter les systèmes informatiques de capacités intellectuelles comparables à celles des humains, de nouveaux systèmes d'aide à la décision médicale, appelés systèmes experts, ont été développés.

\subsection{Les systèmes experts}

Les systèmes experts sont des systèmes à base de connaissances qui visent à simuler le raisonnement des experts engagés dans une démarche décisionnelle. Les connaissances nécessaires à la résolution d'un problème sont ainsi dissociées du raisonnement à mettre en œuvre pour produire la solution. Classiquement un système expert est constitué de 3 modules principaux représentés dans la Figure 5: 
- la base de connaissances qui contient les connaissances médicales théoriques et empiriques nécessaires à la démarche diagnostique ou thérapeutique, au sein d'un domaine d'expertise,

- la base de faits qui représente les caractéristiques du patient pour lequel on cherche à déterminer le diagnostic ou la prise en charge thérapeutique,

- le moteur d'inférences qui articule les connaissances de la base de connaissances pour raisonner sur la résolution du problème posé dans la base de faits.

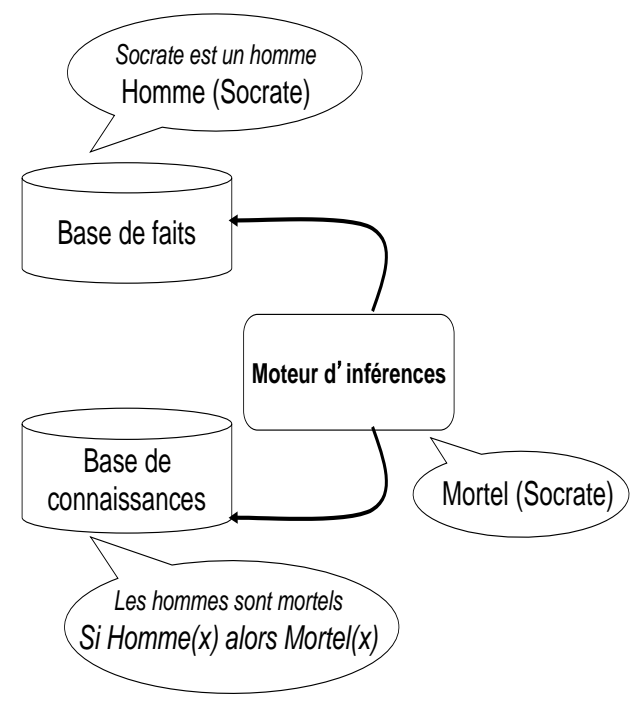

Figure 5: $\quad$ Les 3 modules d'un système expert.

La connaissance experte est décomposée en petites unités homogènes. Chacune d'elle représente alors un fragment élémentaire de l'expertise. Il existe de nombreux langages de représentation de ces connaissances, parmi lesquels les règles de production construites sur le modèle « Si condition Alors action » sont les plus utilisées, par exemple :

\section{Si (Protéinurie > $3 \mathrm{~g} / \mathrm{l}$ et Oedème et Albuminémie < $30 \mathrm{~g} / \mathrm{l})$ Alors syndrome néphrotique.}

Le moteur d'inférences est un programme qui articule ces unités de connaissance pour résoudre le problème posé par un patient donné caractérisé dans la base de faits. Le moteur d'inférences est indépendant de la base de connaissances et de la base de fait. Il construit le raisonnement en chaînant les inférences, essentiellement déductives, ce qui permet de garantir que le résultat obtenu est valide. Pour une nouvelle application médicale du système expert, il suffit de développer une nouvelle base de connaissances, le moteur d'inférences est inchangé.

Mycin est le système expert emblématique de l'informatique médicale (Shortliffe et al., 1975). Développé au début des années 70 à l'université de Stanford par E. Shortliffe, il permettait de fournir une aide au médecin pour le diagnostic et le traitement antibiotique des maladies infectieuses. La base de connaissances comportait près de 500 règles de production assorties de coefficients de certitude. Il était d'autant plus attractif qu'une interface en langage quasi naturel permettait une communication agréable avec l'ordinateur pour le recueil des éléments de description du cas clinique à résoudre (cf. figure 6). Néanmoins, en dépit de ces qualités, le système n'a jamais été utilisé en routine clinique d'une part pour des raisons de responsabilité médico-légale et d'autre part, du fait de considérations éthiques. 


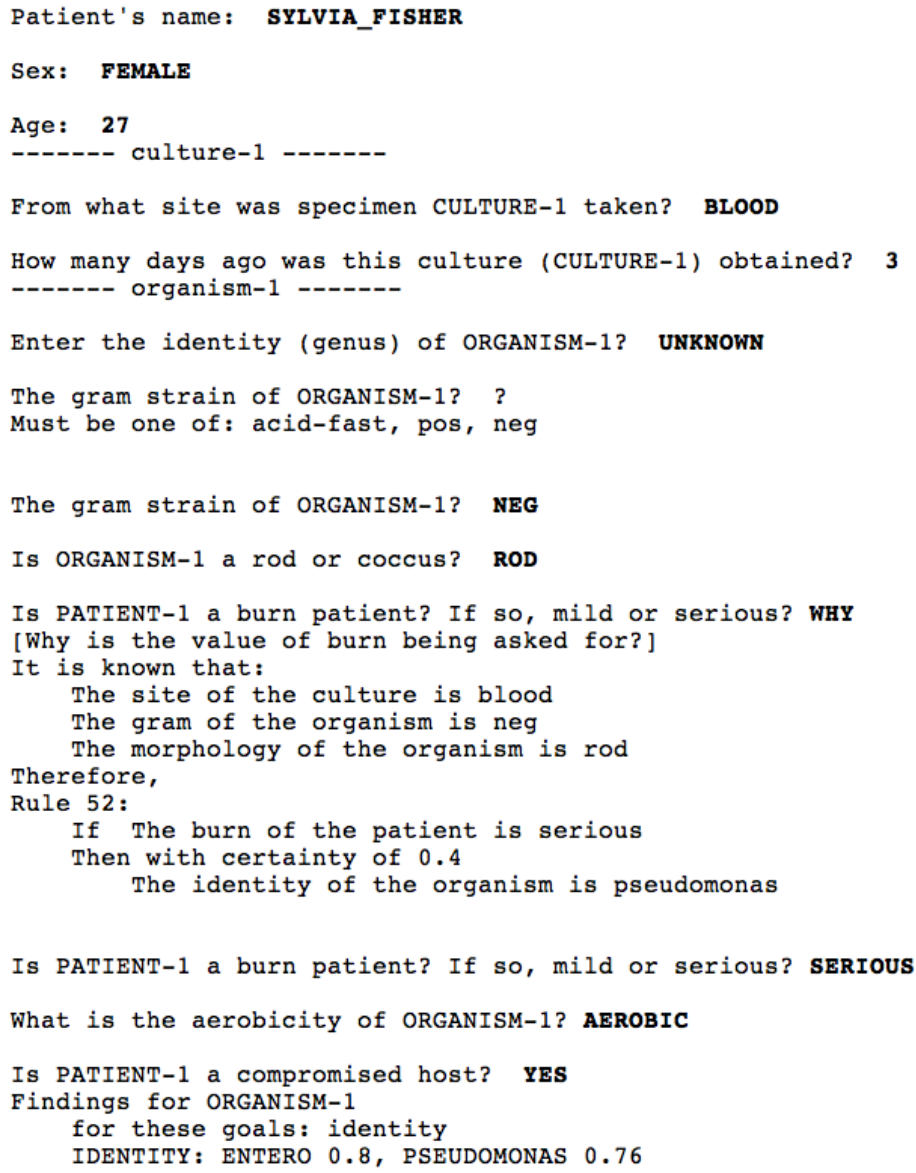

Figure 6: $\quad$ Extrait de l'interface homme-machine en langage naturel avec vocabulaire contrôlé du système Mycin (extrait de http://www-compsci.swan.ac.uk/ csphil/CS345/chapts5-9.pdf, consultation le 27 mai 2017).

Développé au Laboratoire d'Informatique du Massachusetts General Hospital à Boston, DXplain est un autre système expert remarquable (Barnett et al, 1987). Utilisé depuis 1986 et commercialisé depuis 1987, sa base de connaissances a augmenté au fil des ans d'environ 500 maladies dans la première version à plus de 2400 maladies, 5000 critères cliniques et 230000 données. Les utilisateurs entrent des données cliniques dans une zone de texte, à partir desquelles le système cherche les règles médicales qui s'appliquent à ces données dans sa base de connaissances (cf. Figure 7). A l'issue de cette recherche, DXplain propose une liste de diagnostics possibles, en distinguantles diagnostics fréquents et les diagnostics rares. Il peut également poser des questions sur des critères cliniques permettant de discriminer de façon efficace entre les hypothèses diagnostiques retenues. Des liens vers des fiches résumées des maladies et les références MEDLINE correspondantes permettent à l'utilisateur d'accéder à des informations additionnelles sur le diagnostic sélectionné. 


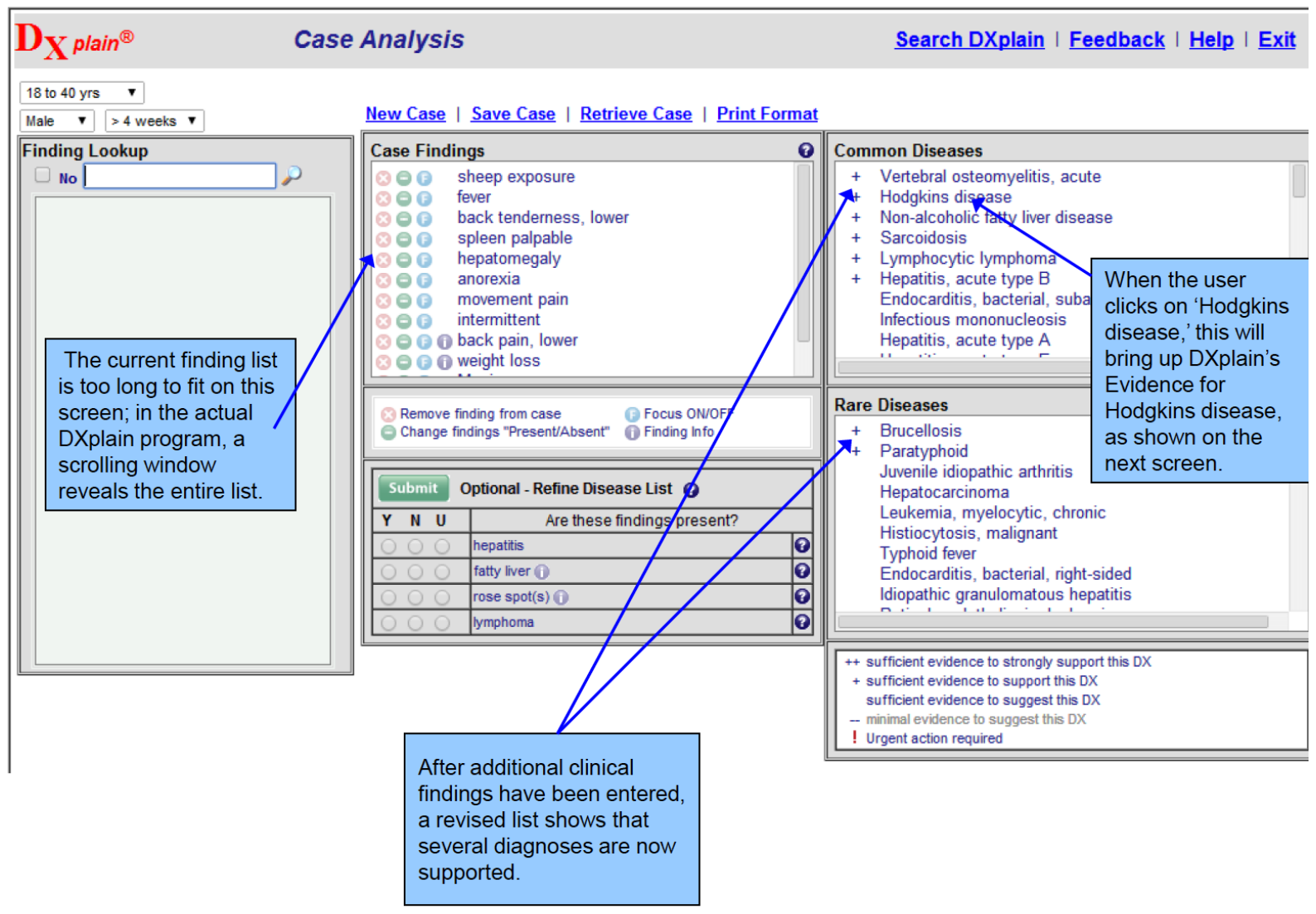

Figure 7: $\quad$ Interface de la démo DXplain accessible à http://dxplain.org/demo2/

Pourtant, malgré un engouement initial, les systèmes experts médicaux ne sont pas devenus des outils de la pratique courante des médecins. Si de nombreux obstacles à leur déploiement peuvent être identifiés, un argument mis en avant par leurs détracteurs était que, du fait du mode de constitution des bases de connaissances, ces systèmes ne traduisaient que l'expertise de leurs concepteurs. Cette expertise n'était pas nécessairement partagée, pouvait être discutable et n'avait pas de légitimité à prescrire ou dicter les pratiques à suivre. Avant qu'un système informatisé ne soit utilisé dans un processus décisionnel, la question de l'origine et de la validité des connaissances sur lesquelles il fonde ses déductions se pose. Cette problématique n'est pas spécifiquement liée aux systèmes experts.

\subsection{Les systèmes de mise en ouvre des recommandations de pratique clinique}

La volonté de s'appuyer sur des connaissances valides pour prendre des décisions s'est concrétisée dans les années 1990 par un courant d'origine anglo-saxonne promouvant une médecine «fondée sur des données probantes » ou evidence-based medicine (EBM, même en français). La pratique de l'EBM consiste à utiliser pour toute prise de décision, de manière rigoureuse, judicieuse et explicite, les dernières données scientifiques, c'est-à-dire celles issues d'une recherche clinique méthodologiquement bien conduite et validés par des publications dans des revues scientifiques, qui constituent l'état de l'art médical (Sackett et al., 1996). Ceci n'exclut pas de tenir compte également de la singularité des patients et d'intégrer leur système de valeurs et leurs préférences. Toutefois, cette démarche idéalisée est difficile à mettre en œuvre en routine. Les médecins ne peuvent pas effectuer une synthèse de l'état de l'art pour identifier les connaissances pertinentes à chaque consultation.

Une partie du problème est résolu par les « recommandations de pratique clinique » (RPC) ou « guides de bonnes pratiques » ou encore «lignes directrices »- les termes anglais correspondantsétantclinical practice guidelines, best practices, clinical pathways. Les RPC sont des documents réalisant une synthèse de l'état de 
l'art sur une problématique médicale donnée. Elles émanent de sociétés savantes, par exemple le GRESEP (Groupe de Réflexion sur la Sclérose En Plaque), ou d'agences nationales comme la Haute Autorité de Santé en France. Ces documents textuels, au style narratif, répertorient des situations cliniques particulières pour lesquelles des prises en charge sont recommandées. Les recommandations sont généralement qualifiées par un grade qui informe le lecteur sur le niveau de preuve des études à l'origine de leur production. Ainsi, les connaissances issues des RCP possèdent a priori un statut plus objectif et transparent qu'une expertise locale ${ }^{3}$. Aussi, il était naturel que les RPC se substituent aux expertises et deviennent les sources des bases de connaissances des nouveaux systèmes experts, rebaptisés «systèmes d'aide à la décision médicale », en anglais Clinical Decision Support Systems, au milieu des années 1990. Débarrassés du handicap lié à la source de l'expertise, les SADM deviennent alors des outils de promotion des bonnes pratiques.

Toutefois, le passage d'une connaissance médicale exprimée dans des textes, avec toutes les nuances permises par la langue naturelle, à une représentation formelle exploitable par des ordinateurs reste une tâche difficile, non totalement résolue (Shiffman et al., 2004). Aussi, entre le texte d'origine et un système automatique d'aide à la décision, de nouvelles approches sont proposées pour structurer le contenu des RPC et permettre un accès facilité, rapide et pertinent, aux recommandations. Développées dès le début des années 2000 dans le contexte du déploiement de l'internet, ces approches que l'on qualifie de documentaires utilisent l'informatique non plus à la manière des systèmes experts pour raisonner sur des connaissances automatiquement exécutables par un ordinateur, mais pour permettre aux cliniciens d'y accéder de manière ciblée dans une démarche de recherche d'information. Parmi les approches documentaires, plusieurs outils ou services existent illustrant différents niveaux de structuration, allant du texte à la base de connaissances entièrement formalisée. Les outils suivants illustrent de manière non exhaustive ces niveaux de structuration de manière croissante.

- UpToDate $^{4}$ (Fox et Moawad, 2003) ou ClinicalKey ${ }^{5}$ (Vardell, 2013) sont des services en ligne payants, fournissant des fiches d'information sur les problèmes de santé (cf. Figure 8). Ces fiches proposent une aide au diagnostic, à la thérapeutique et à l'éducation du patient. Elles sont élaborées par des experts du domaine sur la base de l'état de l'art avec des références aux ressources mobilisées. Le contenu de ces fiches est actualisé en continu. Les fiches restent essentiellement textuelles, elles ne sont donc pas exploitables automatiquement par un système et doivent être lues.

3 Une littérature abondante existe questionnant la validité des RPC au travers des multiples biais potentiels dans la chaîne d'élaboration des recommandations. Ces aspects ne sont pas abordés ici.

4 http://www.uptodate.com/

5 https://www.clinicalkey.com 


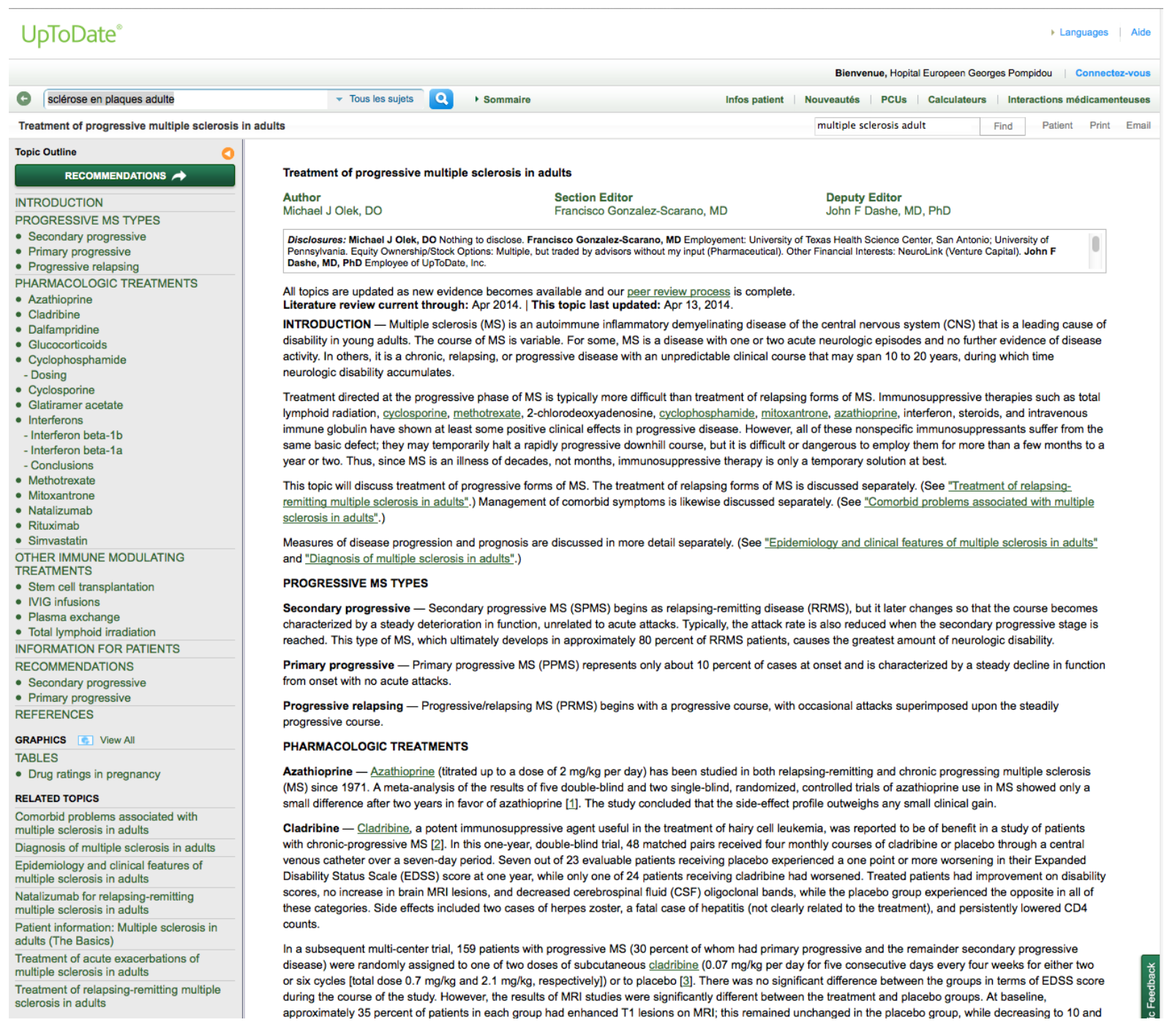

Figure 8: Accès aux recommandations pour la prise en charge thérapeutique de la SEP avec UpToDate (accès au sitehttp://www.uptodate.com/ en cherchant «Multiple sclerosis », le 27 mai 2014)

- Plus structuré, VidalRecos, est un recueil de recommandations proposé par VIDAL, publié sous la forme d'un livre mais également sous la forme d'un site $w^{e} b^{6}$. Pour un ensemble de pathologies, une courte synthèse des RPC existantes, françaises et internationales, est proposée et présentée sous une forme semi structurée. On y trouve des informations textuelles (diagnostic, objectifs de prise en charge, etc.), des arbres décisionnels sous forme de schémas, des liens vers des moteurs de recherche médicaux (cf. Figure 9). Vidal tire partie de sa base de données médicamenteuse pour fournir un lien entre les classes thérapeutiques liées à la pathologie mentionnées dans les RPC et les spécialités médicamenteuses concernées. 


\section{Les Recos > Sclérose en plaques}

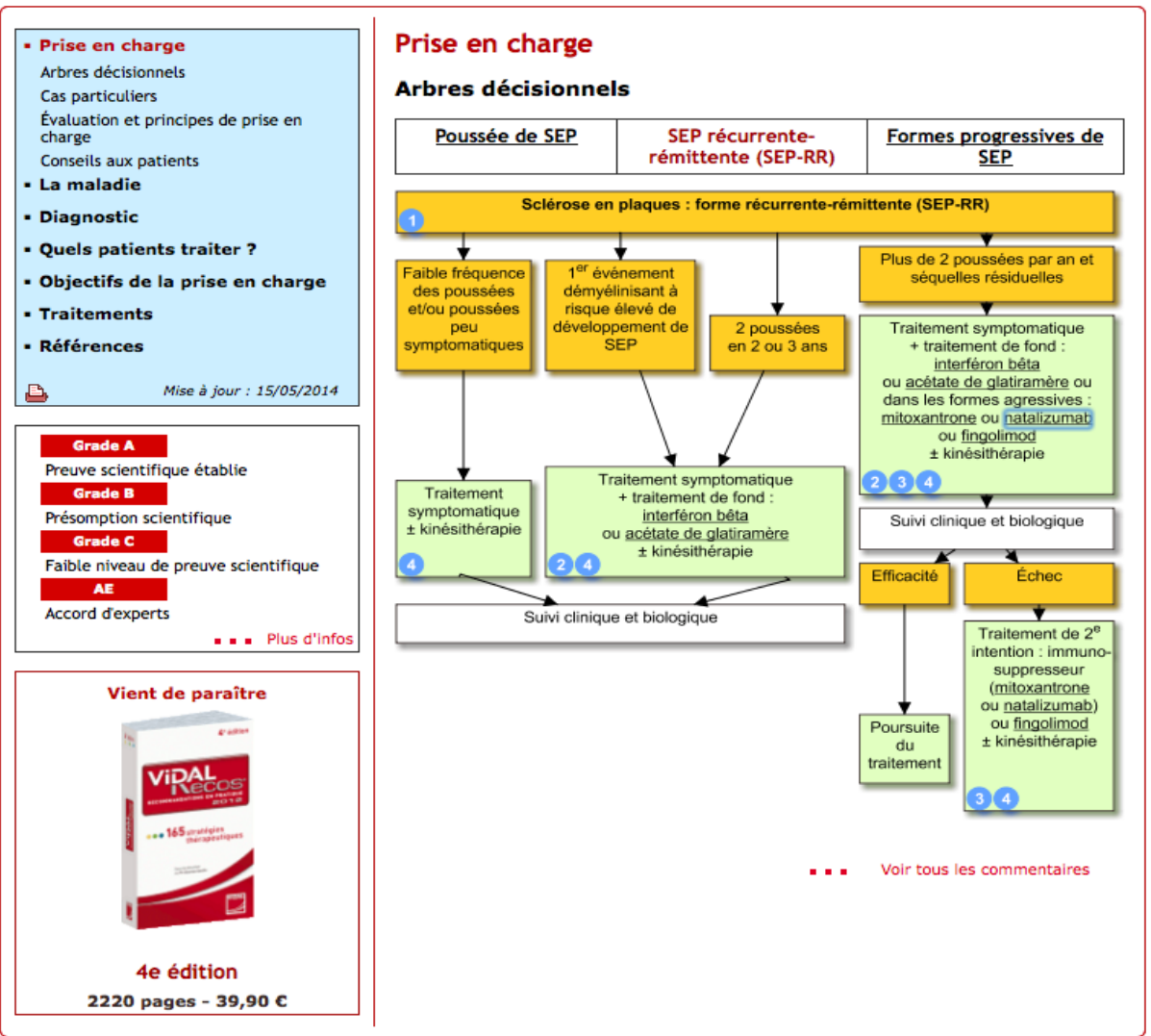

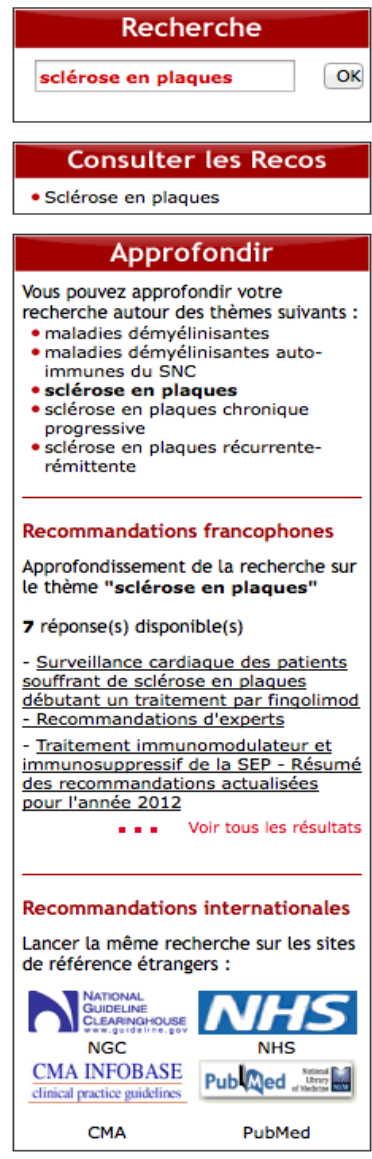

FiTII Nous adhérons aux

Figure 9: $\quad$ Accès aux recommandations pour la prise en charge de la SEP avec VidalRecos (accès au site http://www.vidalrecos.frlen cherchant "sclérose en plaque », le 27 mai 2014)

- Encore plus structuré, les NICE Pathways ${ }^{7}$ proposent une représentation graphique des RPC produites par le National Institute for Health and Care Excellence au Royaume-Uni. Pour chaque sujet traité, la prise en charge recommandée est structurée de manière synthétique sous la forme d'un arbre décisionnel. Certains des noeuds sont cliquables et renvoient à un texte explicatif (cf. figure 10), ou bien à un nouveau sous-arbre décisionnel. L'ensemble forme un hypertexte au sein duquel l'utilisateur navigue pour y trouver les recommandations du NICE qu'il cherche. 


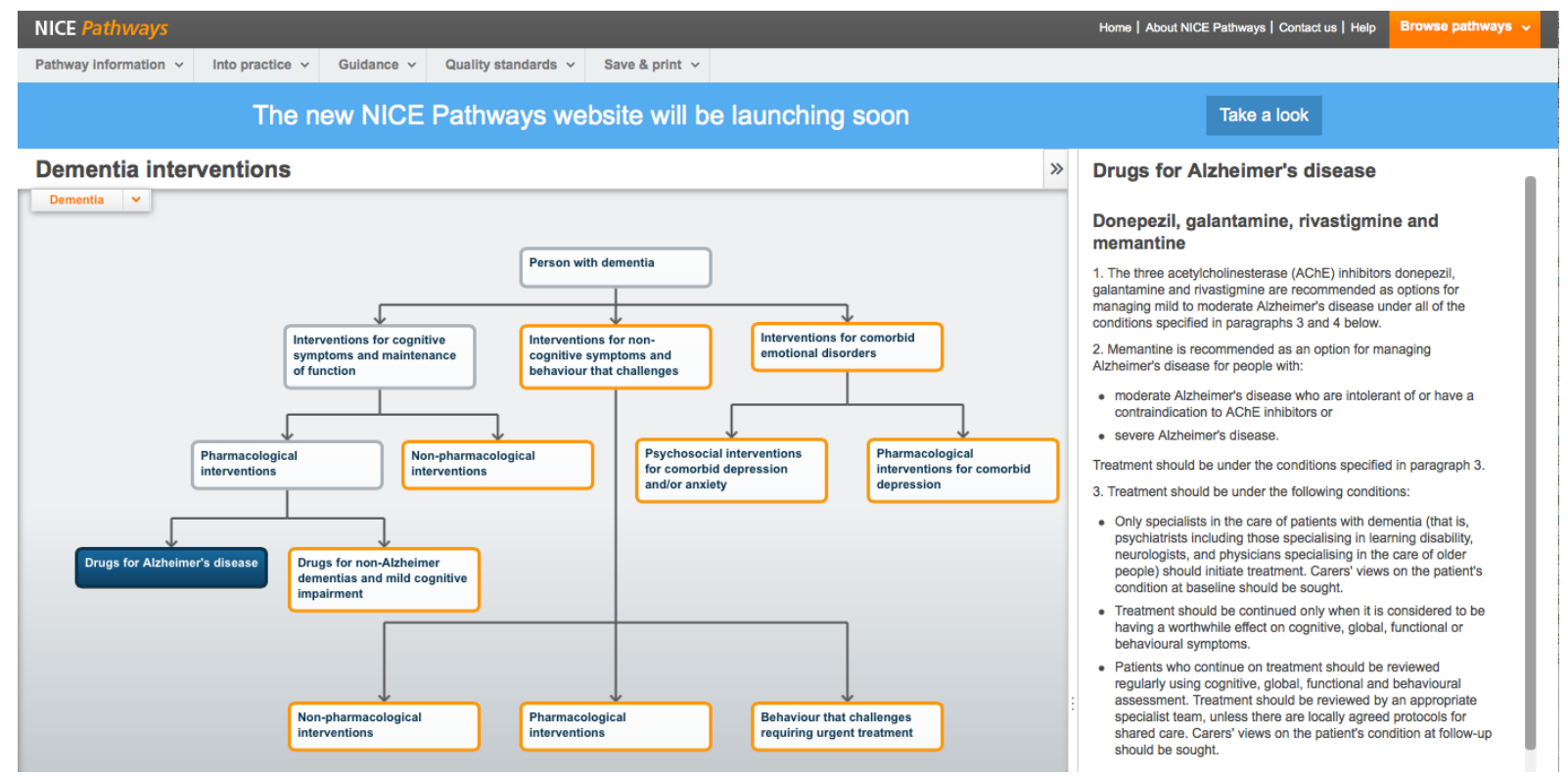

Figure 10: Accès aux recommandations pour la prise en charge d'un patient atteint de démence par navigation dans un arbre décisionnel dynamique. Le clic sur le noud "Traitement médicamenteux de la maladie d'Alzheimer » ouvre uncadre(à droite) contenant un texte explicatif (accès au site http://pathways.nice.org.uk/avec" Dementia»le 27 mai 2014).

- OncoDoc (Séroussi et al., 2001) est un SADM appliqué à la prise en charge thérapeutique des cancers du sein. La base de connaissance est structurée sous la forme d'un arbre de décision dont les nœuds représentent les critères patients et les chemins représentent les profils patients auxquels sont associées au niveau des feuilles de l'arbre de décision les recommandations appropriées. Dans cette approche, la base de connaissance peut-être exploitée de manière documentaire par un utilisateur naviguant dans l'arbre de décision pour caractériser un cas clinique et obtenir les recommandations appropriées (cf Figure 11), ou bien de façon automatique si des données patient codées sont disponibles et peuvent être utilisées pour documenter la navigation. Cette approche a également été utilisée pour modéliser les recommandations de prise en charge thérapeutique de l'HTA, de la dyslipidémie, et du diabète (Séroussi et al., 2014). 


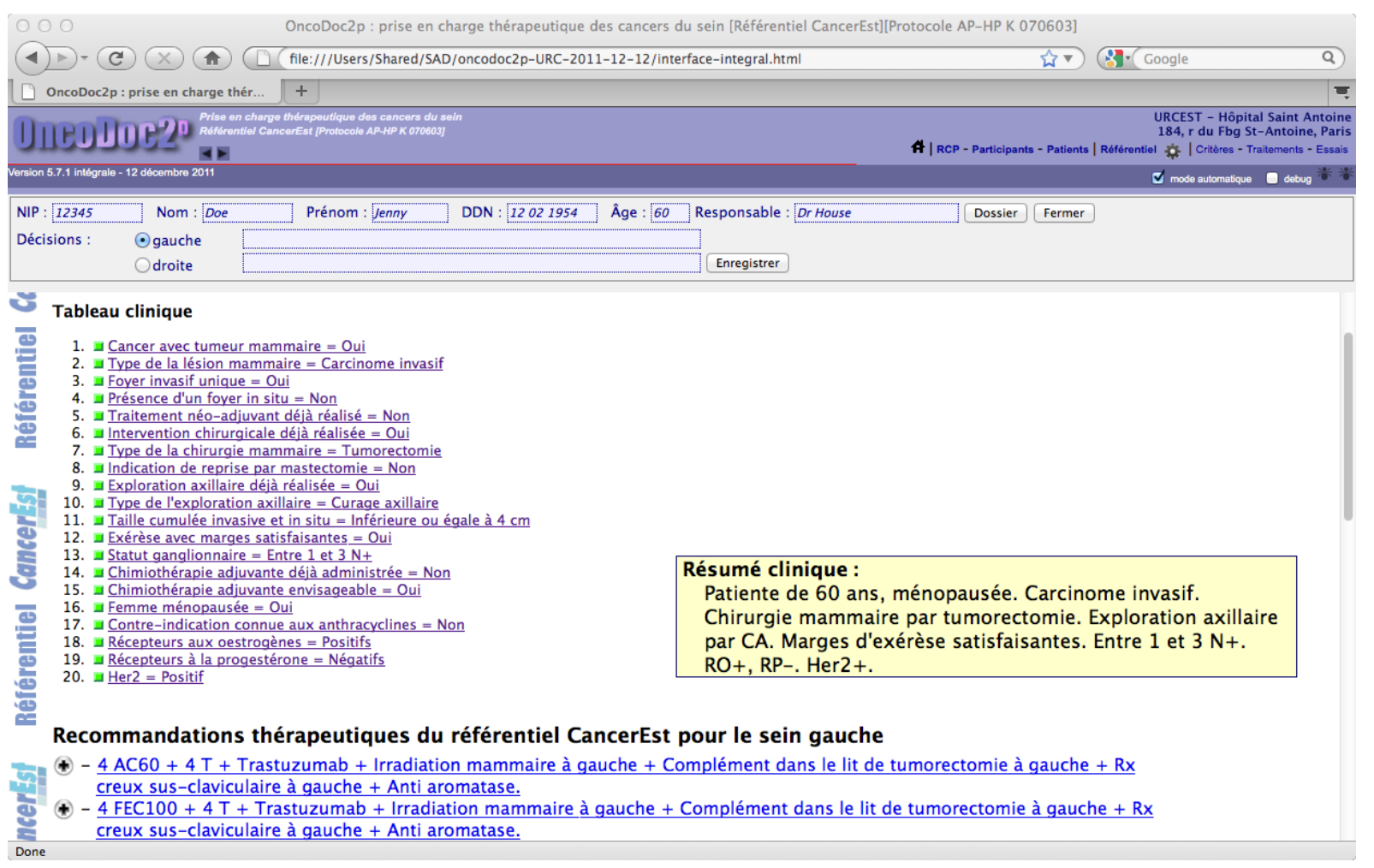

Figure 11 : Ecran de fin de navigation du système OncoDoc2 permettant d'obtenir les recommandations appropriées au profil patient décrit au cours de la navigation et rappelé par le tableau clinique récapitulatif.

\section{Modalités d'exploitation des connaissances et interfaces utilisateurs}

Les bases de connaissances des systèmes d'aide à la décision peuvent être exploitées selon différentes modalités par la structure de contrôle du raisonnement (le moteur d'inférences des systèmes experts). Comme évoqué ci-dessus, deux grands types d'interaction peuvent être distingués selon le degré de structuration des connaissances : les approches documentaires et les approches automatiques.

\subsection{Les approches documentaires}

Dans les approches documentaires, les bases de connaissances sont organisées selon la logique documentaire du texte des RPC dont elles sont issues. C'est le cas des systèmes qui utilisent des bases de connaissances non structurées tels que UpToDate, ClinicalKey, VidalRecos, les parcours du NICEou OncoDoc (mode documentaire). Dans ce cas, le médecin utilisateur du SADM est le médiateur de la recherche d'information. Il navigue au sein de la base de connaissances en suivant des liens dynamiques ce qui lui permet de sélectionner les ressources qui correspondent à la question qu'il se pose pour un patient donné. Il est également à même d'interpréter les réponses proposées et d'identifier ainsi la meilleure décision. Ainsi, dans le cas des approches documentaires, les bases de connaissances ne sont pas opérationnalisées par le système, mais par la lecture de l'utilisateur. L'avantage de ces approches réside dans le fait qu'elles ne dépendent pas de l'existence de données patient codées et dans le fait que le médecin garde le contrôle du raisonnement. Il est actif dans la démarche et engagé dans la recherche des informations qui lui sont nécessaires pour décider. Par contre, ces approches présentent des inconvénients: $(i)$ il faut que le médecin soit conscient d'être en difficulté et recherche effectivement des informations, (ii)le temps mis pour trouver la réponse peut ne pas être compatible avec la durée d'une consultation, d'autant que la recherche peut être infructueuse, la réponse étant souvent « trop spécifique d'un domaine ou d'un patient»(Cimino et al., 2003). Ainsi, un médecin généraliste se pose en moyenne 11 questions par jour ( 2 tous les 3 patients), ne recherche des réponses qu'à $55 \%$ d'entre elles et les obtient dans $40 \%$ des cas (Ely et al., 2005). 


\subsection{Les approches automatiques}

Les approches automatiques répondent à la définition des SADM proposée par Hunt et al. (1998) : «A CDSS is defined as any software ... in which characteristics of individual patients are matched to a computerized knowledge base for the purpose of generating patient-specific assessments or recommendations ». Ainsi, les bases de connaissances doivent être formalisées, les caractéristiques du patient objet de la décision doivent être codées, les deux langages de formalisationdoivent être compatibles. Le raisonnement est alors conduit par une structure de contrôle qui utilise les données du patient pour chercher dans la base de connaissances à quelles situations elles se rapportent. Une fois la ou les situation(s) identifiée(s), les décisions recommandées, qu'elles soient diagnostiques ou thérapeutiques, sont proposées par le SADM. Le raisonnement est le plus souvent déductif et s'appuie sur le chaînage de règles de production. Il peut également s'apparenter à un parcours automatique à travers un graphe de représentation des connaissances du domaine (p. ex. les arbres de décision d'OncoDoc2) jusqu'au nœud qui représente la situation théorique à laquelle le patient correspond et de laquelle l'action (ou les actions) appropriée(s) sont dérivée(s).

Les approches automatiques peuvent être utilisées en mode consultation, elles sont alors utilisées à la demande, comme les approches documentaires, pour obtenir le(s) traitement(s)recommandé(s) ou les diagnostics probables. Elles sont néanmoins le plus souvent utilisées sans l'intervention directe de l'utilisateur et produisent des alertes pour signaler des résultats d'examens anormaux, le rappel d'actions programmées à réaliser, ou pour «critiquer» les prescriptions de l'utilisateur (médicamenteuses, ou d'examens complémentaires) qui ne respectent pas les RPC. Compte tenu du manque de spécificité des alertes, de nombreuses publications ont rapporté une «fatigue aux alertes » conduisant parfois les utilisateurs à «débrancher » le SADM. Ce mode d'interaction est l'objet de travaux pour mieux cibler le contenu et la pertinence des messages transmis aux cliniciens.

Par ailleurs, la qualité du codage de l'état du patient conditionne la qualité de l'aide à la décision. Si le codage du patient présente des erreurs, ou s'il n'est pas exhaustif, la représentation codée du patient,qui est la seule accessible au système, ne correspond pas au profil patient réel et la recommandation générée par le système peut être inappropriée. De plus, lors de l'utilisation automatique d'un SADM, le médecin utilisateur perd le contrôle de la contextualisation des connaissances. Il peut arriver que le patient codé représente alors une caricature erronée de l'état réel du patient dont la singularité est perdue. Ces décalages peuvent se produire en particulier avec les seuils (patient âgé si $>75$ ans, chimiothérapie si taille de la tumeur $>2 \mathrm{~cm}$ etc.) et la mise en œuvre de techniques complexes basées sur la logique floue ne font que reculer le problème sans réellement le résoudre.Dans ce cas, des informations contextuelles non pris en compte par la modélisation de la base de connaissances, et donc inaccessibles au SADM, peuvent conduire le SADM à générer des recommandations que l'utilisateur pourra considérer comme inappropriées.

\subsection{Les approches mixtes}

Les modes d'exploitation des bases de connaissances sont souvent uniquement documentaires ouuniquement automatiques. Pourtant un seul mode de fonctionnement ne répond pas nécessairement à tous les besoins des médecins. Au contraire, ces deux modalités de l'aide à la décision sont en faitcomplémentaires. Par exemple, un médecin peut vouloir prescrire comme il le souhaite, sans avoir à passer par un SADM, quitte à être averti par une alerte automatique en cas de non suivi de recommandations, ou bien rechercher de l'aide en se documentant avant de prescrire le meilleur traitement recommandé. Certains systèmes proposent des approches mixtesoffrant les 2 types de modalités ou les combinant. Ainsi, des données patients codées peuvent permettre d'accéder directement, et de manière ciblée, à des ressources documentaires (transformation des codes patients en codes MeSH et lancement automatique d'une requête Medline) oudes RCP. Ce mode d'accès intermédiaire, fonction du contexte, est mis en œuvre avec les infobuttons utilisés dans les dossiersmédicaux informatisés (Cimino et al., 2005).

\section{Discussion et conclusion}

Au cours des cinquante dernières années, les SADM se sont développés par vague. D’abord centrés sur l'aide au diagnostic, ils ont récemment évolué pour fournir un support à décision thérapeutique, en particulier par la mise en œuvre des RPC. Même si de nombreux outils existent déjà, leur déploiement en pratique reste modeste, en tout cas au niveau du territoire français. Quelques explications peuvent être proposées.

Tout d'abord, les RPC qui constituent les sources de connaissances des SADM sont très critiquées par 
les professionnels de santé (Cabana et al., 1999). En effet, au delà des doutes que certains expriment sur la fiabilité scientifique des contenus (cf. l'actualité récurrente sur les problèmes liés aux conflits d'intérêt des experts), les RPC sont souvent considérées comme non appropriées aux cas particuliers, inutiles pour les cas simples et manquantes pour les cas complexes. Issues des résultats des essais cliniques disponibles sur une thématique donnée, elles sont par nature incomplètes et ne proposent pas de solution de prise en charge pour de nombreux profils cliniques. Supposées s'appuyer sur des données probantes, on constate que peu de recommandations sont effectivement associées à un grade et que la majorité d'entre elles reposent sur des accords professionnels. Enfin, le temps mis pour l'élaboration des RPC (entre 18 et 24 mois) et la fréquence de leur réactualisation (tous les 5 à 10 ans), font que ces documents sont rapidement obsolètes.

Il existe d'autres obstacles à la mise en œuvre des SADM que le contenu des RPC, et on pourra se reporter à la revue de Flottorp et al. (2013) pour une classification des facteurs déterminant les pratiques médicales.En particulier, si l'effet des SADM sur les pratiques est variable, mais globalement positif, avec une amélioration des mesures de performance comme le suivi des recommandations (Jaspers et al., 2011 ; Roshanov et al., 2013), l'impact clinique de l'usage de ces outils reste encore faible. Pour relativiser ces résultats, on pourra objecter que ces conclusions s'appuient sur des publications anciennes ayant testé des SADM encore plus anciens. Mais les choses sont en train de changer avec le développement des objets connectés, et la mise à disposition,notamment du grand public, de service numériques liés à la santé et à la médecine via des applications, en particulier mobiles (sur smartphones et tablettes). Ces applicationsproposent aux professionnels de santé le calcul de scores, la consultation de RPC, l'accès à la littérature, et selon Worster et Haynes (2012), il est à présent aisé d'obtenir des réponses aux questions cliniques qu'un médecin se pose. Desoutils similaires sont développés à destination des patients et plus largement des usagers. Par exemple, sympto-check ${ }^{8}$ et e-docteur ${ }^{9}$ en France permettent au patient d'identifier un diagnostic probable à partir d'un interrogatoire et du recueil de signes et de symptômes à la manière de DXplain. Une application mobile comme «AVC, agir vite » permet à chacun de diagnostiquer une situation d'urgence et d'alerter la SAMU en cas de suspicion d'AVC à travers une procédure formalisée évaluant l'état de conscience, la qualité du discours, les anomalies du bras et du visage.

Aujourd'hui, la manière de concevoir les SADM a évolué et, même s'il ne sont pas encore énormément répandus,ils continuent d'être envisagés comme des instruments d'évaluation des états de santé, de promotion des bonnes pratiques et de personnalisation des soins. Face à la demande actuelle d'efficience et de qualité des soins, leur développementet leur diffusion vont s'amplifier comme en témoigne les incitatifs mis en œuvre à la manière du « Meaningful use » aux Etats-Unis ${ }^{10}$ (rémunération perçue sur la preuve d'usage des technologies de l'information en santé), avec, pour la ville, la rémunération sur objectif de santé publique (ROSP) incluant déjà l'informatisation du cabinet, l'utilisation d'un dossier patient informatisé et le recueil d'indicateurs cliniques, et le programme Hôpital Numérique pour les établissements de santé. Toutefois, la place des SADMne sera ni unique, ni centrale, etdevra se concevoir au sein d'approches multidimensionnelles jouant sur tous les leviers pour permettre le transfert efficace des connaissances, et la promotiondes bonnes pratiques (Grimshaw et al., 2012).

Il est donc vraisemblable que la médecine aura à s'exercer dans un environnement de plus en plus informatisé et connecté oùles SADM joueront des rôles variés. Pour les professionnels de santé, ce seront des outils simplificateurs, garants d'une pratique de qualité. Pour les établissements de santé,ce seront des instruments d'harmonisation des pratiques et de mesure de performance. Utilisés par les patients, les SADM participeront à leur autonomisation, source potentielle d'adhésionaux soins et d'observance.Dans tous les cas,la qualité et la pertinence des messages délivrés par les SADMdépendront des ressources sur lesquelles ils seront adossés, que ce soit les jeux de données, les bases de connaissances, mais aussi les données utilisées pour décrire les patients. Aux développeurs de SADM d'être explicites sur l'origine des ressources utilisées. Aux médecins de s'informer tout en conservant leurs sens critique et clinique afin de tirer parti de ces nouveaux outils dans leurs prises de décision.

\section{Remerciements}

Nous remercions le Dr Olivier Steichen pour sa relecture approfondie et pour ses commentaires de clinicien qui nous ont permis d'améliorer la qualité du document.

\footnotetext{
8 http://www.docteurclic.com/sympto-check/

9 http://www.e-sante.fr/e-docteur/

$10 \mathrm{http} / / / \mathrm{ww}$.healthit.gov/providers-professionals/meaningful-use-definition-objectives
} 


\section{Références}

[1] Barnett GO, Cimino JJ, Hupp JA, Hoffer EP. DXplain. An evolving diagnostic decision-support system. JAMA 1987;258(1):67-74.

[2] Bellows J, Patel S, Young SS.Use of IndiGO individualized clinical guidelines in primary care. J Am Med Inform Assoc 2014;21(3):432-7.

[3] Berner ES. Clinical decision support systems: State of the Art. AHRQ Publication No. 09-0069-EF. Rockville, Maryland: Agency for Healthcare Research and Quality. June 2009.

[4] Cabana MD, Rand CS, Powe NR, Wu AW, Wilson MH, Abboud PA, Rubin HR. Why don't physicians follow clinical practice guidelines? A framework for improvement. JAMA 1999;282(15):1458-65.

[5] Cimino JJ, Meyer M, Lee NJ, Bakken S. Using patient data to retrieve health knowledge.AMIA Annu Symp Proc 2005:136-40.

[6] de Dombal FT, Leaper DJ, Staniland JR, McCann AP, Horrocks JC. Computer-aided diagnosis of abdominal pain. Br Med J 1972;2(5804):9-13.

[7] Ely JW, Osheroff JA, Chambliss ML, Ebell MH, Rosenbaum ME.. Answering physicians' clinical questions: obstacles and potential solutions. J Am Med Inform Assoc 2005;12:217-24.

[8] Flottorp S, Oxman A, Krause J, Musila N, Wensing M, Godycki-Cwirko M, et al. A checklist for identifying determinants of practice: A systematic review and synthesis of frameworks and taxonomies of factors that prevent or enable improvements in healthcare professional practice. Implement Sci 2013; 8(1): 35.

[9] Fox GN, Moawad N. UpToDate: a comprehensive clinical database.J Fam Pract 2003; 52(9): 706-10.

[10] Grimshaw JM, Eccles MP, Lavis JN, Hill SJ, Squires JE. Knowledge translation of research findings. Implement Sci 2012; 7: 50.

[11] Horrocks, A. P. McCann, J. R. Staniland, D. J. Leaper, and F. T. de Dombal. Computer-aided Diagnosis: Description of an Adaptable System, and Operational Experience with 2,034 Cases. Br Med J1972; 2(5804): 5-9.

[12] Hunt DL, Haynes RB, Hanna SE, Smith K. Effects of computer-based clinical decision support systems on physician performance and patient outcomes: a systematic review. JAMA 1998;280(15):1339-46.

[13] Jaspers MW, Smeulers M, Vermeulen H, Peute LW. Effects of clinical decision-support systems on practitioner performance and patient outcomes: a synthesis of high-quality systematic review findings. J Am Med Inform Assoc 2011;18(3):327-34.

[14] Ravdin PM, Siminoff LA, Davis GJ, Mercer MB, Hewlett J, Gerson N, Parker HL. Computer program to assist in making decisions about adjuvant therapy for women with early breast cancer. J Clin Oncol 2001; 19(4): 980-91.

[15] Roshanov PS, Fernandes N, Wilczynski JM, Hemens BJ, You JJ, Handler SM, et al. Features of effective computerised clinical decision support systems: meta-regression of 162 randomised trials. BMJ 2013; 346: f657.

[16] Sackett DL, Rosenberg WM, Gray JA, Haynes RB, Richardson WS. Evidence based medicine: what it is and what it isn't. BMJ 1996;312(7023):71-2.

[17] Séroussi B, Bouaud J, Antoine EC. OncoDoc: a successful experiment of computer-supported guideline development and implementation in the treatment of breast cancer.Artif Intell Med 2001;22(1):43-64.

[18] Séroussi B, Falcoff H, Ertel-Pau V, Raimond V, Bouaud J.Online dissemination of clinical practice guidelines as narrative texts and structured pathways: a case study with the treatment of type 2 diabetes. Stud Health Technol Inform 2014;A paraître.

[19] Shiffman RN, Michel G, Essaihi A, Thornquist E. Bridging the guideline implementation gap: a systematic, document-centered approach to guideline implementation. J Am Med Inform Assoc 2004;11(5) :418-26.

[20] Shortliffe EH, Davis R, Axline SG, Buchanan BG, Green CC, Cohen SN. Computer-based consultations in clinical therapeutics: explanation and rule acquisition capabilities of the MYCIN system. Comput Biomed Res 1975;8(4):303-20.

[21] Vardell E.ClinicalKey: a point-of-care search engine.Med Ref Serv Q 2013; 32(1): 84-92.

[22] Wilson DH, Wilson PD, Walmsley RG, Horrocks JC, de Dombal FT. Diagnosis of acute abdominal pain in the accident and emergency department. Br J Surg 1977;64(4):250-4. 
[23] Worster A, Haynes RB. How do I find a point-of-care answer to my clinical question? CJEM 2012; 14(1): $31-5$.

\section{Auteur correspondant :}

B. Séroussi

Département de Santé Publique

Hôpital Tenon

4, rue de la Chine

75020 Paris, France

brigitte.seroussi@tnn.aphp.fr 